\title{
PROJETO, MONTAGEM E AUTOMAÇÃO DE UM REATOR “AIRLIFT" PARA STRIPPING DE ETANOL E ESTIMATIVA EM TEMPO REAL DA HIDRODINÂMICA E COEFICIENTE DE TRANSFERÊNCIA DE MASSA
}

\author{
V. S. RAMOS ${ }^{1}$ e F.V. da SILVA ${ }^{2}$ \\ ${ }^{1}$ Universidade Estadual de Campinas (Unicamp), Departamento de Engenharia de Sistema \\ Químicos \\ ${ }^{2}$ Universidade Estadual de Campinas (Unicamp), Departamento de Engenharia de Sistemas \\ Químicos \\ E-mail para contato: vtrsantana@gmail.com
}

\begin{abstract}
RESUMO - O desenvolvimento da biotecnologia tem causado um impulso em pesquisas acadêmicas e na implantação de bioprocessos na indústria, como o desenvolvimento de reatores conhecidos como airlifts. Estes são provenientes de modificações no design de colunas de bolhas, e constituem de uma coluna preenchida com um líquido, que possui circulação induzida ao aumentar-se a vazão do gás alimentado em sua base. Nestes reatores ocorre um elevado contato gás-líquido, com elevadas transferências de massa e calor, no entanto, sem a necessidade da utilização de agitadores mecânicos, que podem proporcionar perda considerável de biomassa por cisalhamento e contaminação. Assim, este trabalho possui como principais objetivos o projeto, a montagem e a automação de um reator airlift de tubos concêntricos, de modo a obter em tempo real a fração de gás retido e a velocidade de circulação do líquido, e ainda estimar o coeficiente volumétrico de transferência de massa no sistema composto por solução aquosa de etanol e ar, aonde separa-se o etanol do meio através do arraste pelo gás carbônico presente no ar injetado (gas stripping).
\end{abstract}

\section{INTRODUÇÃO}

Têm-se notado atualmente um aumento da utilização de bioprocessos na indústria, estes costumam ser realizados em reatores tanque agitados, no entanto, a utilização destes reatores resultam em perdas consideráveis de biomassa, devidas principalmente ao alto cisalhamento provocado por choques contra a hélice, e também pela presença de impurezas acumuladas em tal componente, que proporcionavam contaminação do meio como consequência.

Diante da necessidade de novas alternativas para os bioprocessos desenvolveram-se novos equipamentos, entre eles a coluna de bolhas, nos quais observou-se que na alimentação um gás na base de uma coluna preenchida previamente com um líquido ocorre um intenso contato gáslíquido, com elevadas taxa de transferência de massa e calor, e também mistura da solução sem a utilização um de agitador mecânico. Diante dessas vantagens estes equipamentos tornaram-se uma alternativa interessante para os processos fermentativos e outros bioprocesos tradicionais, 
principalmente para os que necessitam de elevada demanda por oxigênio dissolvido. As colunas de bolhas possuem também maior facilidade de limpeza e de construção e consequentemente de menor custo de operação e de fabricação.

Uma classe particular deste tipo de equipamento é estudada neste trabalho, trata-se dos airlifts, que se diferenciam das colunas de bolhas pela presença de uma recirculação induzida do líquido. Os airlifts surgiram como consequência direta da inserção de um tubo concêntrico ou de uma chicana na parte interior das colunas, tais inserções proporcionaram assim duas regiões distintas na coluna, e provocam uma diferença de densidade entre a região alimentada pelo ar, duto de transporte (riser), e o duto de recirculação (downcomer), esta diferença de densidade é a força motriz responsável pela circulação do líquido. Conforme aumenta-se a vazão de gás alimentado no reator observa-se três regimes hidrodinâmicos predominantes. O regime I é caracterizado pela ausência de gás no duto de recirculação, o regime II é caracterizado pela presença de bolhas de gás estagnadas no duto de recirculação, e o regime III é identificado pela visualização da circulação de bolhas entre os dutos de alimentação e de recirculação. Observou-se também transferências de massa e calor maiores do que as encontradas anteriormente nos reatores de tanque agitado e coluna de bolhas.

\section{MATERIAIS E MÉTODOS}

\subsection{Dimensionamento do Airlift}

De acordo com o observado no trabalho de Heijnen et al. (1997) em operações em escala industrial somente o regime de completa circulação de gás possui importância, assim optou-se em dimensionar um reator airlift com volume de aproximadamente 331 , superior aos geralmente adotados em escalas de laboratório e capaz de proporcionar a operação do reator no regime III. Notou-se que no trabalho de Chisti et al., (1993) que o emprego de uma região de separação, através de uma modificação simples na região superior da coluna, provoca uma melhora do desempenho do equipamento, tal modificação foi considerada no projeto do airlift utilizado neste trabalho. Na Figura 1 abaixo pode-se observar um desenho esquemático do sistema experimental, pode visualizar-se no interior do airlift a presença de tubos concêntricos e com região de separação no topo da coluna (extensão do diâmetro). 


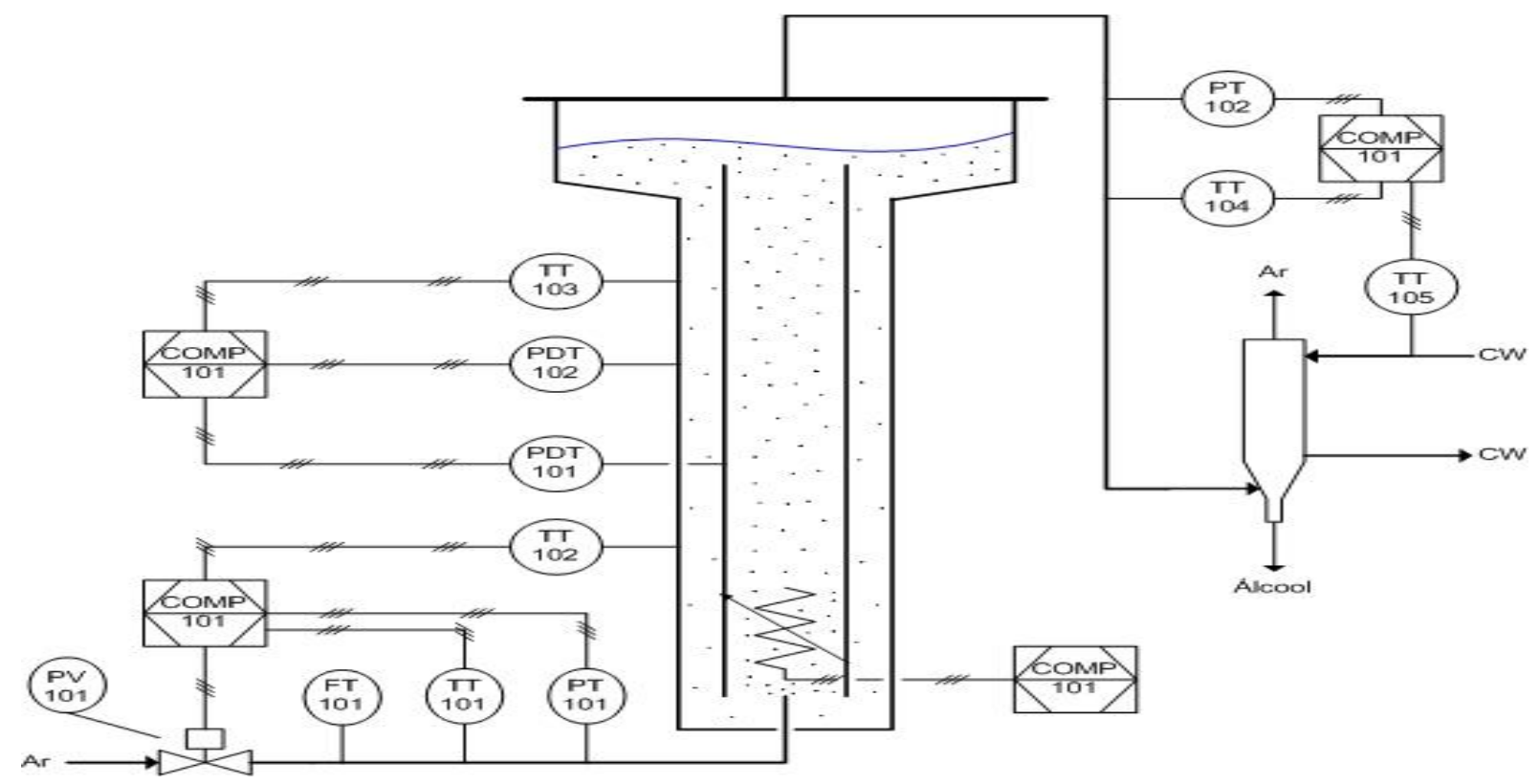

Figura 1 - Desenho esquemático do airlift e sistema experimental

Na Tabela 1 a seguir apresenta-se as dimensões de projeto do airlift utilizado neste trabalho.

Tabela 1 - Dimensões de projeto do airlift

\begin{tabular}{|c|c|c|c|c|c|c|}
\hline $\mathrm{H}_{\mathrm{T}}(\mathrm{cm})$ & $\mathrm{L}_{\mathrm{TC}}(\mathrm{cm})$ & $\mathrm{L}_{\mathrm{S}}(\mathrm{cm})$ & $\mathrm{D}_{\mathrm{T}}(\mathrm{cm})$ & $\mathrm{D}_{\mathrm{R}}(\mathrm{cm})$ & $\mathrm{D}_{\mathrm{S}}(\mathrm{cm})$ & $\mathrm{A}_{\mathrm{D}} / \mathrm{A}_{\mathrm{R}}(\mathrm{cm})$ \\
\hline 190 & 160 & 24,5 & 16 & 11,2 & 30 & 1,04 \\
\hline
\end{tabular}

\subsection{Instrumentação e Equipamentos Complementares}

Aquisição de dados: software LabVIEW ${ }^{\circledR}$.

Sensores de temperatura: sensores/transmissores de temperatura (Novus®).

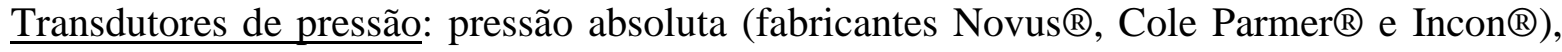
pressão diferencial (fabricante Smar®). Festo®.

Válvula de controle: válvula reguladora de pressão proporcional VPPE-3-1-1/8-2-010-E1,

Válvulas de bloqueio e válvula de retenção: válvulas esfera (fabricantes MGA ${ }^{\circledR}$, IVM $^{\circledR}$ e Sfera ${ }^{\circledR}$ ), válvula de retenção classe 125/200 do fabricante MIPEL®. Tigre®.

Linha de ar: tubulação flexível de borracha (Himaflex®), tubos e conexões rígidas de PVC,

Compressor: modelo MSV 40, fabricante Schulz ${ }^{\circledR}$. 
Resistência elétrica e variador de potência: resistência tubular de potência $2000 \mathrm{~W}$, Thermo Soluções Montagens Industriais Ltda, variador de potência, modelo MTPS-1242-20A, Loti Lorando Tecnologia Industrial Ltda..

Condensador, banho de refrigeração e bomba: condensador Friedrich, Tec Labor ${ }^{\circledR ;}$ banho termostático, modelo III,Fanem ${ }^{\circledR}$ Ltda; bomba centrífuga modelo AC-2CP-MD, March Mfg. Inc..

\subsection{Parâmetros hidrodinâmicos e transferência de massa}

Para a obtenção da taxa de aeração (gas hold-up) no airlift desenvolvido foi utilizada a Equação 1 a seguir, obtida pela realização de um balanço de momento no duto de transporte ou no duto de recirculação, metodologia proposta por Furigo (1986) e também por Carvalho (2001).

$$
\varepsilon_{D, R}=1-\frac{\Delta P_{D, R}}{\rho_{L} g \cdot L}
$$

O valor da velocidade de circulação do líquido que percorre o downcomer do airlift pode ser obtido pelo método do traçador aquecido (hot tracer method) conforme o trabalho de Sijacki et al. (2010). Serão obtidos sinais de temperatura em função do tempo conforme a Figura 2 abaixo:
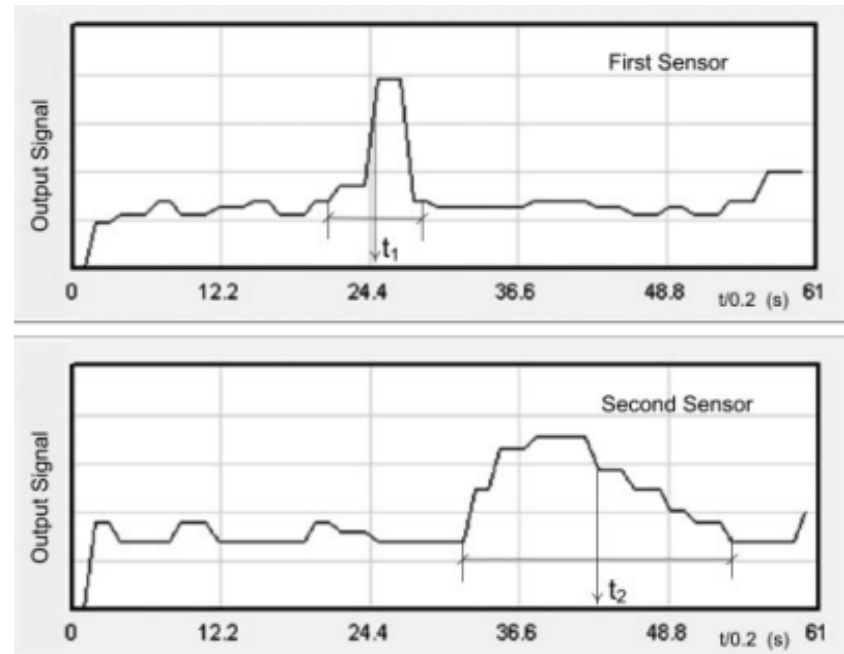

Figura 2 - Método do traçador aquecido (Sijacki et al., 2010).

Da Equação 2 abaixo utilizada em conjunto com curvas similares as observadas na Figura 2 calcula-se a velocidade de circulação do líquido:

$$
Y \overline{\bar{T}} \frac{Z}{\left(t_{2}-z_{1}\right)-\Delta x}
$$


Para obter-se o coeficiente volumétrico de transferência de massa no processo de stripping de etanol optou-se por seguir a metodologia proposta por Furigo (1986), através desta chega-se a Equação 3 abaixo, em tal trabalho encontram-se as hipóteses consideradas:

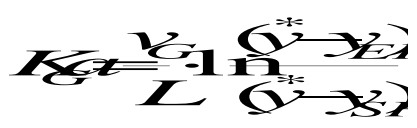

Os dados de equilíbrio necessários para resolução da Eq. (4.17) serão calculados com base no modelo UNIQUAC, os parâmetros binários utilizados neste modelos são obtidos em Gmehling et al. (1991).

\section{RESULTADOS}

Através de uma análise da literatura chega-se a conclusão que os parâmetros que possuem influência relevante na transferência de massa do etanol da solução líquida para o ar são a concentração de etanol, temperatura do meio, vazão de ar alimentado e desnível entre o topo do duto central e a altura do líquido presente no airlift. Deste modo planejou-se experimentos conforme a Tabela 2 e obteve-se os resultados conforme a Tabela 3 e Figuras 3 e 4:

Tabela 2 - Experimentos realizados

\begin{tabular}{|c|c|c|c|c|}
\hline & $\begin{array}{c}\text { g etanol/g total } \\
\text { no airlift }(\%)\end{array}$ & Treator $\left({ }^{\circ} \mathrm{C}\right)$ & Vlíquido $(\mathrm{l})$ & Vsgás $(\mathrm{cm} / \mathrm{s})$ \\
\hline E-01 & 7,5 & 25 & 30,64 & 31,6 \\
\hline E-02 & 16 & 25 & 30,64 & 31,6 \\
\hline E-03 & 8 & 35 & 30,64 & 31,6 \\
\hline E-04 & 16 & 35 & 30,64 & 31,6 \\
\hline E-05 & 7,2 & 25 & 32,64 & 31,6 \\
\hline E-06 & 16 & 25 & 32,64 & 31,6 \\
\hline E-07 & 7,5 & 35 & 32,64 & 31,6 \\
\hline E-08 & 15 & 35 & 32,64 & 31,6 \\
\hline E-09 & 8,6 & 25 & 30,64 & 45,17 \\
\hline E-10 & 16 & 25 & 30,64 & 45,17 \\
\hline E-11 & 8 & 35 & 30,64 & 45,17 \\
\hline E-12 & 16 & 35 & 30,64 & 45,17 \\
\hline E-13 & 7 & 25 & 32,64 & 45,17 \\
\hline E-14 & 15 & 25 & 32,64 & 45,17 \\
\hline
\end{tabular}




\begin{tabular}{|c|c|c|c|c|}
\hline E-15 & 8 & 35 & 32,64 & 45,17 \\
\hline E-16 & 15 & 35 & 32,64 & 45,17 \\
\hline E-17 & 13 & 30 & 31,64 & 40,26 \\
\hline
\end{tabular}

Tabela 3 - Resultados experimentais da transferência de massa

\begin{tabular}{|c|c|c|c|}
\hline & $\begin{array}{c}\text { g etanol/g total } \\
\text { condensado(\%) }\end{array}$ & Qcondensado $(\mathrm{ml} / \mathrm{s})$ & $\mathrm{K}_{\mathrm{G}} \mathrm{a}(\mathrm{ml} / \mathrm{s})$ \\
\hline E-01 & 9,5 & 0,013 & 0,00106 \\
\hline E-02 & 25 & 0,017 & 0,00154 \\
\hline E-03 & 19 & 0,028 & 0,0039 \\
\hline E-04 & 48 & 0,05 & 0,00897 \\
\hline E-05 & 11 & 0,012 & 0,0012 \\
\hline E-06 & 21 & 0,014 & 0,00109 \\
\hline E-07 & 12 & 0,028 & 0,00275 \\
\hline E-08 & 40 & 0,039 & 0,00627 \\
\hline E-09 & 13,8 & 0,024 & 0,00171 \\
\hline E-10 & 22 & 0,019 & 0,00111 \\
\hline E-11 & 13,5 & 0,033 & 0,00257 \\
\hline E-12 & 27 & 0,043 & 0,00296 \\
\hline E-13 & 10 & 0,024 & 0,00152 \\
\hline E-14 & 21 & 0,027 & 0,00154 \\
\hline E-15 & 13 & 0,038 & 0,00263 \\
\hline E-16 & 32 & 0,058 & 0,0051 \\
\hline E-17 & 20 & 0,022 & 0,00163 \\
\hline & & & \\
\hline & & & \\
\hline & 21 & 0 & \\
\hline
\end{tabular}

Através de uma comparação na Tabela 2 entre o par de experimentos 1-3 e uma análise da Tabela 3 chega-se a conclusão de que o coeficiente de transferência de massa aumenta com o aumento da temperatura, pois na temperatura de $35^{\circ} \mathrm{C}$ encontrou-se um valor de $\mathrm{K}_{\mathrm{G}}$ a maior do que o encontrado a $25^{\circ} \mathrm{C}$, sendo que as outras variáveis são mantidas na mesma faixa, observa-se comportamento semelhante nos pares de experimentos 2-4, 5-7, 6-8, 9-11, 10-12, 13-15 e ainda 14-16. De modo semelhante à análise da temperatura observou-se através de uma comparação entre o par de experimentos 1-2 que o $\mathrm{K}_{\mathrm{G}}$ a aumenta com o aumento da concentração, este comportamento repetiu-se nos pares 3-4, 7-8, 11-12, 13-14, 15-16, nos pares 5-6 e também 9-10, o valor de $\mathrm{K}_{\mathrm{g}}$ a diminuiu com o aumento da concentração, tal divergência no comportamento pode ser devido aos efeitos da tensão superficial e coalescência. 
O efeito da velocidade superficial pode ser observado ao comparar-se os pares de experimentos 1-9, 2-10, 3-11, 4-12, 5-13, 6-14, 7-15 e ainda 8-16, da Tabela 3 conclui-se que o $\mathrm{K}_{\mathrm{G}} \mathrm{a}$ aumentou nos pares 1-9, 5-13 e em 6-14, e diminuiu em 2-10, 3-11, 4-12, 7-15 e também em 8-16. A influência do desnível foi verificada nos pares de experimentos 1-5, 2-6, 3-7, 4-8, 9-13, 10-14, 11-15 e 12-16. A transferência de massa aumentou nos pares 1-5, 10-14, 11-15 e em 12-16 e diminuiu em 2-6, 3-7, 4-8, e ainda em 9-13.

A maior transferência de massa foi encontrada no experimento 4, evidenciada pelos maiores valores da fração mássica de etanol no condensado e do $\mathrm{K}_{\mathrm{G}} \mathrm{a}$, neste experimento o airlift foi alimentado com 30,64 1 de solução $16 \%$ de etanol em fração mássica, temperatura de $35^{\circ} \mathrm{C}$, e velocidade superficial de gás de $31,6 \mathrm{~cm} / \mathrm{s}$, ou seja, nos maiores valores de concentração, temperatura do meio e desnível (menor volume), e no menor valor de velocidade superficial do gás. No experimento 8 também encontrou-se um valor superior de fração mássica de etanol e coeficiente de transferência de massa, este experimento difere do experimento 4 apenas pela redução do desnível proporcionado de acordo com o aumento do volume da solução alimentada.

Pode-se observar através da Tabela 4 abaixo que tanto a velocidade de circulação do líquido tanto a fração de gás retido aumentam com o aumento da velocidade de circulação do líquido. $\mathrm{O}$ trabalho vem sendo continuado de forma a avaliar-se a hidrodinâmica nos outros desníveis considerados, visto que tal parâmetro tem influência considerável na transferência de massa, conforme observado anteriormente.

Tabela 4: Influência da velocidade do gás na taxa de aeração e na velocidade de circulação, (32,64 1, desnível 3mm)

\begin{tabular}{|c|c|c|}
\hline Vsgás $(\mathrm{cm} / \mathrm{s})$ & $\in$ (adimensional) & Vlíq $(\mathrm{cm} / \mathrm{s})$ \\
\hline 31,6 & 0,421 & 42,379 \\
\hline 40,26 & 0,485 & 43,403 \\
\hline 45,17 & 0,516 & 43,747 \\
\hline
\end{tabular}

\section{NOMENCLATURA}

$\mathrm{A}_{\mathrm{D}}$ - área do duto de recirculação (downcomer)

$\mathrm{A}_{\mathrm{R}}$ - área do duto de transporte (riser)

$\mathrm{D}_{\mathrm{R}}$ - diâmetro do duto de transporte (riser)

$\mathrm{D}_{\mathrm{S}}$ - diâmetro do separador

$\mathrm{D}_{\mathrm{T}}$ - diâmetro da coluna

$\mathrm{H}_{\mathrm{T}}$ - altura total do airlift (da base da coluna ao topo do separador)

$\mathrm{K}_{\mathrm{G}} \mathrm{a}$ - coeficiente volumétrico de transferência de massa.

$\mathrm{L}$ - distância na tomada de pressão diferencial

$\mathrm{L}_{\mathrm{S}}$ - comprimento do separador

$\mathrm{L}_{\mathrm{TC}}$ - comprimento do tubo concêntrico

$\mathrm{L}_{12}$ - distância entre os sensores de temperatura

$\mathrm{V}_{\mathrm{LD}}$ - velocidade de circulação do líquido

$\mathrm{g}$ - aceleração da gravidade $(\mathrm{m} / \mathrm{s} 2)$ 
$\mathrm{v}_{\mathrm{G}}$ - velocidade superficial do gás $(\mathrm{m} / \mathrm{s})$.

$\mathrm{t}$ - instante de tempo de passagem do traçador

$\mathrm{y}^{*}$ - fração molar de etanol no equilíbrio entre as fases

YER - fração molar de etanol da fase gasosa na entrada do riser

$\mathrm{y}_{S R}$ - fração molar de etanol da fase gasosa na saída do riser

$\Delta \mathrm{P}_{\mathrm{R}, \mathrm{D}}$ - pressão diferencial do $\operatorname{riser}(\mathrm{R})$

$\Delta \mathrm{t}$ - atraso entre os sinais gerados

$\varepsilon$ - taxa de aeração (gas hold-up)

$\rho_{\mathrm{L}}-$ massa específica da fase líquida

\section{REFERÊNCIAS}

CARVALHO, E. Desenvolvimento de modelo hidrodinâmico para reatores air-lift. Dissertação de Mestrado. Faculdade de Engenharia Química, Universidade Estadual de Campinas (Unicamp), Campinas, 2001.

CHISTI, Y.; MOO-YOUNG, M. Improve the performance of airlift reactors. Chemical Engineering Progress, v. 89(3), p. 38-45, 1993.

FURIGO Jr., A. Características hidrodinâmicas e de transferência de massa em contadores "airlift". Tese de Mestrado. Faculdade de Engenharia Química, Universidade Estadual de Campinas (Unicamp), Campinas, 1986.

GMEHLING, J.; ONKEN, U.; ARLT, W.; GRENZHEUSER, P.; WEIDLICH, U.; KOLBE, B.; RAREY, J. Vapor-Liquid Equilibrium Data Collection. Vol 1 (1), 1991.

HEIJNEN, J. J.; HOLS, J.; LANS, H. L. J. M.; LEEUWEN, H. L. J. M. A simple hydrodynamic model for the liquid circulation velocity in a full-scale two-and-three-phase internal airlift reactor operating in the gas recirculation regime. Chemical Engineering Science, v. 52(15), p. 2527-2540, 1997.

SIJACKI, I. M.; TOKIC, M. S.; KOJIC, P. S.; PETROVIC, D. L.; TEKIC, M. N.; DJURIC, M. S.; MILOVANCEV, S. S. Sparger type influence on the hydrodynamics of the draft tube airlift reactor with diluted alcohol solutions. Industrial \& Engineering Chemistry Research, v. 50, p. 3580-3591, 2011. 